\title{
Doble jornada laboral y percepción de la salud en mujeres: investigación cualitativa
}

\author{
Double working day and perception of health in women: qualitative investigation
}

Nadia Musarella $a^{a, b}$, Vilda Disacacciatti ${ }^{a, c, d}$

\begin{abstract}
Resumen
Introducción. El trabajo ocupa un lugar clave en la vida de la mujer, y a su vez comprende varias dimensiones. Dos de ellas son el trabajo doméstico no remunerado (TDNR) e invisibilizado, y el trabajo productivo remunerado (TPR) fuera de la casa. El objetivo de esta investigación fue comprender cómo es la percepción de salud en la mujer actual de clase media urbana con doble jornada laboral y cómo ella jerarquiza su TPR.

Métodos. Investigación con enfoque cualitativo realizada en un hospital privado universitario de la Ciudad de Buenos Aires, mediante entrevistas individuales a mujeres adultas y alfabetas de 30 a 60 años de edad, seleccionadas a través del método de bola de nieve a partir de sus médicos de cabecera. Se utilizaron fichas demográficas estructuradas y entrevistas en profundidad. Se registraron datos personales y prácticas médicas realizadas en los últimos años. Se realizó un análisis de contenido.

Resultados. Entrevistamos a 47 mujeres, la mayoría con hijos y la mitad, a cargo de las tareas domésticas en su hogar. Ninguna de ellas reconoció la tarea doméstica (TDNR) como trabajo, $76 \%$ refirió no realizar controles habituales de salud, aunque más del $90 \%$ hizo las prácticas preventivas recomendadas para su edad. Las mujeres entrevistadas expresaron diferentes significados atribuidos por ellas al TPR como independencia, desarrollo personal, mejoría en autoestima, calidad de vida, y sociabilidad. Sin embargo, ante su ausencia en el hogar se mostraron ambivalentes y con sentimientos de culpabilidad.

Conclusiones. Este estudio permite reconocer que las mujeres con trabajo TDNR y TPR valoran positivamente a este último, pero se cuestionan que éste les quite tiempo a su participación en el ámbito familiar. Esto resulta en una mayor autoexigencia y perpetúa la invisibilidad del TDNR. En relación al autocuidado, sienten que no realizan sus cuidados médicos, pero tienen buenos indicadores de prácticas preventivas. Quizás sea necesario propiciar la reflexión acerca del rol que tiene el TPR en su bienestar.
\end{abstract}

\begin{abstract}
Introduction. Work occupies a key place in the life of women, and in turn, comprises several dimensions. Two of them are unpaid domestic work (UDW) and paid productive work (PPW) outside the home. The objective of this research was to understand how the perception of health is in the current urban middle-class woman with a double working day and how she prioritizes her PPW.

Methods. Research with a qualitative approach carried out in a private university hospital in the City of Buenos Aires, through individual interviews with adult and literates women aged 30 to 60 , selected through the snowball method from their general practitioners. Structured demographics and in-depth interviews were used. Personal data and medical practices carried out in recent years were recorded. A content analysis was performed.

Results. We interviewed 47 women, the majority with children and half of them, in charge of housework in their home. None of them recognized housework (UDW) as work, $76 \%$ reported not doing regular health checks, although more than $90 \%$ did the recommended preventive practices for their age. The women interviewed expressed different meanings attributed by them to the PPW such as independence, personal development, improvement in self-esteem, quality of life, and sociability. However, in their absence at home, they were ambivalent and guilty.

Conclusions. This study allows us to recognize that women with UDW and PPW value the latter positively, but question whether it takes time away from their participation in the family environment. This results in increased self-demand and perpetuates the invisibility of the UDW. In relation to self-care, they feel that they do not perform their medical care, but have good indicators of preventive practices. It may be necessary to encourage reflection on the role of the PPW in their well-being.
\end{abstract}

Palabras clave: Mujeres, Mujeres Trabajadoras, Salud de la Mujer, Inequidad de Género. Keywords: Women, Working women, Women's Health, Gender Inequality.

Musarella N, Discacciati V. Doble jornada laboral y percepción de la salud en mujeres: investigación cualitativa. Evid Actual Pract Ambul. 2020;23(3):e002046.

\footnotetext{
a Servicio de Medicina Familiar y Comunitaria, Hospital Italiano de Buenos Aires.

b Departamento de Medicina, Instituto Universitario Hospital Italiano de Buenos Aires. nadia.musarella@hospitalitaliano.org.ar

c Comité de Bioética, Hospital Italiano de Buenos Aires.

d Departamento de Salud Pública, Instituto Universitario Hospital Italiano de Buenos Aires. vilda.discacciati@hospitalitaliano.org.ar
} 


\section{Introducción}

A lo largo de la historia de la humanidad se ha reflexionado sobre el rol de la "mujer trabajadora", especialmente respecto de lo sucedido durante los periodos de profundas transformaciones económicas, sociales y políticas, y también sobre la repercusión de dichos cambios en la cotidianeidad femenina, más allá del aporte económico de este colectivo a la sociedad.

Previo a la era industrial y tanto en Europa como en América, había mujeres trabajadoras urbanas y rurales. Entrando al siglo $\mathrm{XIX}$, y como describe Scott ${ }^{1}$ "la cuestión de la mujer trabajadora" en el marco de revolución industrial, comienza a ser percibida como problemática y a cobrar visibilidad. Surgen los cuestionamientos sobre la compatibilidad entre la femineidad y el trabajo asalariado (trabajo productivo remunerado: TPR), y sobre la influencia del trabajo en el cuerpo de la mujer y sus consecuencias en el ámbito doméstico. Dicho de otra forma, un encuadre de argumentos opuestos entre hogar y trabajo, maternidad y trabajo asalariado, femineidad y productividad. Por ejemplo, se sostenía que era conveniente que las mujeres trabajaran fuera de su casa y en forma asalariada, solamente durante períodos cortos, que se retiraran después de casarse o de haber tenido hijos, y que sólo debían volver a desarrollar un trabajo remunerado fuera del ámbito doméstico (TPR) en el caso de que su marido no pudiera mantener a la familia.

Las mujeres comenzaron a concentrarse en empleos mal pagos y no calificados, que reflejaban la prioridad de la labor doméstica, entendida como "problema" en un mundo en el que el trabajo asalariado y las responsabilidades familiares se habían convertido en empleos a tiempo completo, espacial y sexualmente diferenciados. Comenzó a cristalizarse la separación entre hogar y trabajo, con diferencias entre mujeres y hombres, lo que legitimó e institucionalizó las diferencias de género como base de la organización social. Vale destacar que desde finales del siglo XIX comenzó a ocurrir un desplazamiento desde el trabajo de las mujeres en el servicio doméstico hacia los denominados "empleos de cuello blanco" en oficinas gubernamentales, empresas y compañías de seguros, que comenzaron a requerir habilidades diferentes, como son las habilidades comunicacionales de lecto-escritura y las específicas de cada oficio. Sin embargo, y como continúa sucediendo en la actualidad, para la contratación de mujeres ya se establecía una edad límite y era frecuente que se obstaculizaran los matrimonios entre personas de la misma empresa, procurándose mantener un plantel con mano de obra barata y homogénea.

En 1906, las mujeres constituían en Francia más del $40 \%$ de la fuerza de trabajo de cuello blanco ${ }^{2}$ y comenzaron a plantearse debates en relación a sus condiciones de trabajo. Por ejemplo, William Gaskell describió cómo los pechos de las mujeres trabajadoras resultaban no aptos para amamantar a sus hijos mientras trabajaban en la fábrica, mientras otros argumentaban la incompatibilidad entre mujeres y maquinaria. Incluso también algunos entendían que era inmoral que las mujeres desarrollaran trabajo pesado o estuvieran expuestas al rudo lenguaje masculino.

Por otro lado, se acusaba al trabajo fuera del hogar como causa de enfermedades de las mujeres. Por ejemplo, Paul Lafargue propuso en 1892 una política de permiso por maternidad y un estipendio diario a partir del cuarto mes de embarazo y hasta el final del primer año posterior al parto ${ }^{3}$.

Respecto de la invisibilidad del TDNR, en Gran Bretaña, el censo de 1881 fue el primero que excluyó de la categoría de trabajo a las faenas domésticas de las mujeres. Si bien, antes de ese momento, mujeres y hombres habían presentado niveles similares de actividad económica ${ }^{4}$, luego de esta exclusión censal, la tasa de actividad femenina quedó reducida a la mitad, lo que contribuyo a afianzar la concepción antitética entre domesticidad y productividad, y a que las amas de casa comenzaran a no ser consideradas trabajadoras. Más aún, cuando percibían salarios por realizar tareas como costura en su casa, para los encuestadores no significaba un auténtico trabajo. El predominio de este tipo de interpretaciones fue conduciendo a que gran parte del trabajo de las mujeres fuera ignorado en las estadísticas oficiales, convirtiéndose en un trabajo invisible, que aún persiste en el modelo económico-social vigente, que ignora y subestima la importancia del TDNR. Esta interpretación condujo que aún hasta nuestros días, en el imaginario social el concepto de trabajo haya quedado restringido a actividades que proporcionan una remuneración visible, dentro de la lógica del intercambio mercantil.

Respecto del TDNR y de crianza, el modelo socio-cultural vigente continúa asignando las prácticas domésticas a las mujeres. Sin embargo, se asume que ellas valoran tener un trabajo remunerado bajo los supuestos de que este mejora su autoestima y satisface sus deseos de independencia frente al varón.

Por otro lado y como venimos desarrollando, la sociedad construye identidades a partir de una serie de expectativas sobre las "conductas apropiadas" de hombres y mujeres, basadas en estereotipos de género ${ }^{5}$; y si bien las relaciones entre hombres y mujeres fueron cambiando profundamente ${ }^{6}$, Wainerman ${ }^{7}$ documentó que tres cuartos de los hombres participan nada o muy poco en las actividades cotidianas del cuidado de la casa. También existe evidencia de que en el terreno privado los hombres continúan manifestando desorientación, desconcierto y resistencia frente a la velocidad de los cambios que atraviesan las relaciones de género ${ }^{8}$. Se trata de un proceso que sucede con mucha lentitud e insuficiente compromiso, como lo reflejan algunas giros linguísticos que denominan "colaboración" a las tareas domésticas realizadas por el hombre, como si este fuera alguien de fuera del hogar que ayuda a la persona que tiene la verdadera responsabilidad por dichas tareas hogareñas ${ }^{9}$ : la mujer.

\section{Justificación de esta investigación}

Por eso, y dado que la economía globalizada ha obligado a que la mujer desarrolle tareas remuneradas para contribuir al sostén económico de su familia, -mientras todavía persisten los estereotipos de género que continúan asignándole la principal responsabilidad sobre el trabajo hogareño y de crianza-, se establece una situación particular de desarrollo de una "doble jornada" o "doble carga" laboral "invisible" que obliga a las mujeres a asumir tanto el TPR, como el TDNR, lo que para Federici ${ }^{10}$ conduce a que se deterioren sus condiciones de vida y a que desarrollen con mayor frecuencia ansiedad $u$ otros problemas de la salud mental.

Este estudio se propuso comprender cómo las mujeres logran combinar esta doble tarea y cómo perciben que esta situación repercute en su salud; y también explorar si esta percepción es diferente en mujeres de distintas generaciones, influidas por diferentes estereotipos de género. Su objetivo primario fue comprender en mujeres con doble carga laboral de diferentes generaciones, cómo influye la suma de sus trabajos -el remunerado y el no remunerado- en su bienestar, en la percepción de su salud y en su autocuidado.

Como objetivos secundarios, nos propusimos: a) explorar sus prioridades personales en relación a su actividad laboral y al significado y la valoración que ellas le atribuyen a su trabajo, y también respecto de la distribución de roles (p. ej. tareas domésticas) en las familias actuales de clase media; b) explorar la presencia de potenciales adicciones (tabaco y alcohol) y también cómo afrontan algunas condiciones crónicas como la obesidad y la hipertensión, cuyo manejo exitoso depende del mantenimiento de un estilo de vida saludable; y c) explorar sus prioridades personales respecto del cuidado de su salud e indagar sobre la realización de algunas prácticas preventivas (pesquisa de cáncer de cérvix, mamario y colorrectal). 


\section{Materiales y métodos}

\section{Diseño y población}

Se realizó un estudio descriptivo, de corte transversal con enfoque cualitativo, mediante la realización de entrevistas en profundidad $^{11}$. Se seleccionó una muestra por conveniencia de mujeres alfabetizadas de 30 a 60 años de edad, atendidas en un Hospital Universitario Privado de la Ciudad Autónoma de Buenos Aires.

Los criterios de exclusión fueron: 1) estar recibiendo antipsicóticos y/o haber estado internada en el Servicio de Psiquiatría durante los dos años previos, 2) diagnóstico de enfermedad oncológica, 3) divorcio o separación reciente durante los seis meses previos, 4) desocupación reciente (durante los seis meses previos), 5) paridad reciente (seis meses previos), 6) pérdida de embarazo deseado durate los tres meses previos. Fueron excluidas estas mujeres ya que dichas situaciones podrían haber desviado la temática tocada durante la entrevista o afectar la sensibilidad de las entrevistadas.

A los fines de optimizar la convocatoria, esta se realizó a través del método de la bola de nieve, a partir de varios médicos de familia de diferentes Centros de salud ${ }^{12}$. Cada vez que estos identificaban mujeres que cumplían con los criterios de inclusión, les preguntaban si estaban de acuerdo en que las contactara la investigadora principal para invitarlas a participar.

\section{Métodos para la recolección de los datos}

Para la recolección de datos demográficos de las participantes se completaron fichas estructuradas en las que se consignaron sus datos personales, sus horarios de trabajo, el contexto familiar y la realización de prácticas preventivas.

Se utilizó la entrevista en profundidad como técnica de recolección de datos en el marco de la investigación cualitativa, debido a que este abordaje proporcionaría información rica, variada y complementaria, explorando las experiencias individuales.

Se usó una guía de pautas de entrevista, pero se favoreció la espontaneidad del discurso de las participantes. Los temas abordados fueron el estado general de salud, la valoración y el significado del trabajo remunerado y no remunerado, así como su influencia sobre los cuidados de la salud.

El número de entrevistas realizadas estuvo sujeto al criterio de saturación del discurso, siguiendo el muestreo teórico ${ }^{13}$. Como método de registro, se tomaron notas durante las entrevistas y todas fueron grabadas. La mayoría de las entrevistas fueron realizadas en consultorios, sin que hubiera interrupciones, y unas pocas, en ámbitos naturales como el lugar de trabajo de las entrevistadas. Fueron grabadas en audio. Se respetó en todo momento la anonimidad de las participantes, archivándose las fichas y los audios con un código de números y letras ${ }^{14}$. Se organizó una carpeta de archivos en formato WAV (WaveForm Audio File), compartida por las investigadoras dentro de un servicio de alojamiento de archivos multiplataforma en la nube, con acceso mediante usuario y contraseña. La desgrabación y transcripción de las entrevistas a un formato MS Word fue realizada por la investigadora principal.
Tabla 1. Características demográficas y del cuidado de la salud de las participantes entrevistadas en este estudio $(\mathrm{N}=47)$

\begin{tabular}{|c|c|c|}
\hline Características & & $N(\%)$ \\
\hline Edad (años) & $\begin{array}{l}30 \text { a } 39 \\
40 \text { a } 49 \\
50 \text { a } 60\end{array}$ & $\begin{array}{l}17(36) \\
15(32) \\
15(32) \\
\end{array}$ \\
\hline Educación & $\begin{array}{l}\text { Primaria } \\
\text { Secundaria } \\
\text { Terciaria } \\
\text { Universitaria }\end{array}$ & $\begin{array}{c}1(2) \\
4(8) \\
11(20) \\
31(66)\end{array}$ \\
\hline Estado civil & $\begin{array}{l}\text { Soltera } \\
\text { Casada } \\
\text { Viuda } \\
\text { Separada }\end{array}$ & $\begin{array}{c}20(42) \\
13(28) \\
1(2) \\
13(28)\end{array}$ \\
\hline Sin hijos & & $21(45)$ \\
\hline Con hijos $(\mathrm{N}=26)$ & $\begin{array}{l}\text { hijo } \\
2 \text { hijos } \\
3 \text { hijos } \\
\end{array}$ & $\begin{array}{c}11(23) \\
14(30) \\
1(2) \\
\end{array}$ \\
\hline Convivientes & $\begin{array}{l}\text { Vive sola } \\
\text { Con hijos } \\
\text { En pareja } \\
\text { Con pareja e hijos } \\
\text { Otros }\end{array}$ & $\begin{array}{c}13(28) \\
9(19) \\
6(13) \\
18(38) \\
1(2)\end{array}$ \\
\hline Ocupación & $\begin{array}{l}\text { Profesional independiente } \\
\text { Empleada permanente } \\
\text { Empleada por contrato } \\
\text { Cuentapropista } \\
\text { Trabajadora temporaria } \\
\text { Trabajadora a domicilio } \\
\text { Ama de casa }\end{array}$ & $\begin{array}{l}16(34) \\
18(38) \\
5(11) \\
6(13) \\
1(2) \\
1(2) \\
0(0) \\
\end{array}$ \\
\hline $\begin{array}{l}\text { Cobertura de } \\
\text { salud }\end{array}$ & $\begin{array}{l}\text { Obra Social } \\
\text { Medicina Prepaga }\end{array}$ & $\begin{array}{l}15(32) \\
32(68)\end{array}$ \\
\hline $\begin{array}{l}\text { Estado de salud } \\
\text { autorreportado }\end{array}$ & $\begin{array}{l}\text { Muy bueno } \\
\text { Bueno }\end{array}$ & $\begin{array}{l}13(28) \\
34(72)\end{array}$ \\
\hline $\begin{array}{l}\text { Consulta ante } \\
\text { problema de salud }\end{array}$ & $\begin{array}{l}\text { Automedicación } \\
\text { Remedios caseros } \\
\text { Farmacéutico } \\
\text { Hospital } \\
\text { Médico particular / Obra Social }\end{array}$ & $\begin{array}{c}8(17) \\
1(2) \\
1(2) \\
19(40) \\
18(38)\end{array}$ \\
\hline $\begin{array}{l}\text { Control periódico } \\
\text { de salud }\end{array}$ & $\begin{array}{l}\text { Realiza } \\
\text { No realiza }\end{array}$ & $\begin{array}{l}11(23) \\
36(77)\end{array}$ \\
\hline
\end{tabular}

\section{Análisis cualitativo}

Se realizó el análisis de contenido, mediante los siguientes pasos: elaboración de un sistema de categorías a partir de la dimensiones planteadas; codificación desde el árbol planteado inicialmente, abierto para incluir categorías nativas, e iteración del proceso de identificación de conceptos, categorías, grupos y patrones del contenido de las entrevistas.

Se completó una planilla de MS Excel con las unidades de texto codificadas y se elaboraron mapas conceptuales, que fueron discutidos y problematizados para concluir con el proceso interpretativo y llegar a una narrativa final ${ }^{15,16}$ 


\section{Aspectos éticos}

En todos los casos, luego de aceptar la invitación a participar y antes de comenzar la entrevista, las participantes otorgaron por escrito su consentimiento informado ${ }^{17}$. El protocolo de este trabajo fue aprobado por el Comité de Ética de Protocolos de Investigación del Hospital Italiano de Buenos Aires.

\section{Resultados}

\section{Características de las participantes}

El trabajo de campo se desarrolló durante seis meses, durante 2013. Fueron entrevistadas 47 mujeres de 30 a 60 años de edad. De acuerdo a las notas de campo tomadas por de las investigadoras, el clima durante las entrevistas fue cálido, tranquilo y de confianza.

Todas las mujeres entrevistadas tenían TPR y habían recibido algún tipo de educación formal. La mayoría de ellas había completado estudios universitarios y tenían hijos. Además, la mayoría contaba con un seguro de salud (medicina prepaga) y consideran "bueno" a su estado de salud (ver Tabla 1).

En relación con el cuidado de su salud, si bien $77 \%$ de las participantes referían no realizar controles periódicos de salud (ver Tabla 1), $96 \%$ había tenido al menos un control de la tensión arterial (TA) durante los dos años anteriores, y todas habían realizado un papanicolaou (PAP) al menos una vez en su vida (ver Tabla 2).

Tabla 2. Pautas de autocuidado de la salud reportadas por las participantes entrevistadas en este estudio. Notas: PAP: Papanicolaou; TA: tensión arterial; Ns/Nc: No sabe o no contesta.

\begin{tabular}{|l|l|c|}
\hline $\begin{array}{l}\text { Prácticas pre- } \\
\text { ventivas }\end{array}$ & Indicadores de control de salud & N (\%) \\
\hline $\begin{array}{l}\text { Prevención } \\
\text { cardiovascular }\end{array}$ & $\begin{array}{l}\text { Realizó algún registro de TA en los } \\
\text { Realiza actividad física }\end{array}$ & $45(96)$ \\
& Fuma & $19(40)$ \\
& Consume alcohol a diario & $11(23)$ \\
& Realizó PAP alguna vez en la vida & $47(15)$ \\
cáncer de cérvix & Último PAP hace 3 años & $43(91)$ \\
& PAP hace 5 o más años & $3(7)$ \\
& Ns/Nc & $1(2)$ \\
\hline $\begin{array}{l}\text { Prevención del } \\
\text { cáncer de mama }\end{array}$ & Realizó mamografía & $27(57)$ \\
\hline $\begin{array}{l}\text { Prevención del } \\
\text { cáncer de colon }\end{array}$ & Realizó alguna prueba de rastreo & $13(28)$ \\
& Le ofrecieron realizar el rastreo pero & $4(8)$ \\
no lo hizo & $30(64)$ \\
\hline
\end{tabular}

La mayoría de las mujeres respondió que no realizaba actividad física en forma regular (60\%), que no fumaba (77\%), y que no bebía alcohol (85\%) (ver Tabla 2). Cinco mujeres (11\%) refirieron haber sido víctimas de violencia de género alguna vez . Alrededor de la mitad de las participantes refirieron ser la única persona de la familia a cargo de las tareas domésticas (47\%) (ver Tabla 3). El tiempo promedio semanal dedicado al TDNR fue 13,4 horas, y al TPR, 43,5 horas (ver Tabla 4).
Tabla 3. Contribución al trabajo doméstico no remunerado (TDNR) reportado por las mujeres entrevistadas en este estudio

\begin{tabular}{|l|c|}
\hline $\begin{array}{l}\text { ¿Con quién comparte la responsabilidad del trabajo } \\
\text { doméstico? }\end{array}$ & $\mathrm{N}(\%)$ \\
\hline Sola & $22(47)$ \\
\hline Con la pareja e hijos & $10(21)$ \\
\hline Con personal permanente & $4(9)$ \\
\hline Con personal temporario & $11(23)$ \\
\hline
\end{tabular}

Tabla 4. Duración de la doble jornada laboral (trabajo productivo remunerado y trabajo doméstico no remunerado) comunicada por las mujeres entrevistadas en este estudio. Nota: * Promedio de horas semanales de trabajo

\begin{tabular}{|l|c|c|}
\hline Grupos etarios & $\begin{array}{l}\text { Productivo remune- } \\
\text { rado* }^{*}\end{array}$ & $\begin{array}{l}\text { Doméstico no } \\
\text { remunerado* }^{*}\end{array}$ \\
\hline 30 a 39 años & 44,5 & 14,3 \\
\hline 40 a 49 años & 44,9 & 11,7 \\
\hline 50 a 60 años & 41,1 & 14,1 \\
\hline Total & 43,5 & 13,4 \\
\hline
\end{tabular}

\section{Percepciones de las mujeres entrevistadas}

Organizamos el reporte narrativo del análisis interpretativo en ocho ejes, con la finalidad de facilitar la lectura del trabajo: TPR, TDNR, articulación entre ambos (TPR-TDNR), cuidados de salud, tiempo de recreación, disponibilidad para consultar al médico, ambivalencias en cuanto a la exigencia percibida, significado del trabajo.

Cabe señalar que los ejes y las dimensiones señalados no funcionan de manera fragmentada, sino por el contrario, todos los temas tratados se encuentran interrelacionados entre sí y se enhebran desde la biografía y el contexto de cada una de las mujeres que prestaron su voz a esta investigación. Los referentes empíricos más relevantes se presentan a continuación de los hallazgos en cada uno de los temas.

\section{Trabajo productivo remunerado}

Diferentes componentes del discurso reflejan que, para estas mujeres, el hecho de trabajar representa una actividad productiva, pero también es una de las "puertas de salida" de sus casas. Lo consideran como una actividad que las mantiene activas, y desde ese lugar sienten que les "da vida". Para ellas, salir de la casa y trabajar fuera del hogar implica capacitarse, formarse, actividades que les brindan gratificación y bienestar personal. Manifiestan que tener un trabajo fuera del hogar tiene las ventajas de propiciar la construcción de auto-seguridad, aportar sustento económico, y mejorar su autoestima y su estado de ánimo. Además, tener trabajo fuera de la casa es una oportunidad de apertura en la esfera social, de mejora en la comunicación y de favorecimiento del desarrollo de vínculos interpersonales. Por otro lado, la mayoría concuerda en que realizar TPR requiere de un esfuerzo extra, que les ocupa mucho tiempo y esto lleva a que la mayor parte del tiempo se sientan agotadas. Destacan principalmente que las jornadas laborales les resultan extensas y piensan que ellas, por su condición de género, tienen menos oportunidades de crecer laboralmente que los hombres. No obstante, la mayoría de las mujeres entrevistadas refiere que el balance es positivo a favor de ejercer algún TPR fuera del hogar. 
"[....] no sirvo para estar ociosa. Me entretengo siempre con algo. Si no estoy trabajando, en casa estoy haciendo cosas o dibujo, o le coso cosas a las chicas. Para mí el trabajo es, es transformador [...]"

(55 años, empleada)

"[...] no puedo vivir sin trabajar, trabajé todo la vida, no puedo estar sin trabajar [...]"

\section{(41 años, profesional)}

"[...] actualmente mucho estrés, estoy muchas horas de trabajo, mucha presión $[\ldots] "$

(30 años, profesional)

\section{Trabajo doméstico no remunerado}

La mayoría de las participantes encuentra al rol femenino como el eje principal del hogar: la mujer es la que plancha, limpia, cocina, se encarga del cuidado de los hijos, la "real ama de casa" y la responsable efectiva del TDNR. Aquellas mujeres que tienen varios hijos, cuando ambos miembros de la pareja trabajan fuera de la casa y en aquellas familias que cuentan con sostén económico suficiente, comparten parte de ese trabajo doméstico con personal contratado, principalmente temporario y de sexo femenino. Aquellas mujeres con dificultades económicas para contratar personal doméstico cuentan con la ayuda de familiares, principalmente las abuelas de sus hijos. Las mujeres con hijos adolescentes, refieren que muchas veces comparten con ellos tareas del hogar.

En algunas familias, quizás las más conservadoras, se continúa considerando al hombre como el proveedor del hogar y quien maneja el dinero. Es el varón quien trabaja fuera de la casa para ser el principal sostén familiar. En este tipo de familias, las mujeres se encargan de realizar a solas el trabajo TDNR. Consideran que el hombre quiere que la mujer lo sirva, por lo tanto no realiza ninguna tarea doméstica, y en el caso de realizar alguna tarea en el hogar, se trata por lo general de algún arreglo estructural de la casa. En el caso de realizar alguna tarea habitual, como por ejemplo, lavar la cocina, esto es percibido como una "colaboración" con ellas.

En otras familias, por lo general más jóvenes o más recientemente constituidas, a las que pertenecen las mujeres de 30 a 40 años de edad, se puede observar una mayor homogeneidad en cuanto a la división de las tareas del hogar. Estas diferencias se pueden asociar con los cambios generados en la sociedad actual en la que ambos miembros de la pareja trabajan fuera de la casa.

"[...] tengo que pedir que hagan las cosas. Siento que no las hacen por motus propio, que me cuesta mucho que sientan eso. Mi hija se ocupa de su cuarto. Al que más le hago concesiones es a mi marido, él no limpia [...]"

(55 años, empleada)

"[... por lo general pasa todo por mi, ... me quedé con un solo trabajo, soy la que más está en la casa, el resto lo hacen mis hijas y por ahí mi marido, sus cosas, su cuarto, mi marido algún mandado, el resto yo [...]"
"[...] yo y la empleada hacemos las cosas del hogar. Mi marido y mis niños no ayudan con nada [...]"

(37 años, profesional)

"[...] lo que se está ganando hoy es el respeto por los roles y entender que hay una evolución, hoy la mujer tiene en el mercado de trabajo un rol más significativo y eso no puede estar vinculado a la manera de ver a la mujer como en el pasado vinculada a los cuidados del hogar [...]"

(40 años, profesional)

Articulación entre trabajo reproductivo y el trabajo productivo

La mayoría de las entrevistadas concuerdan en que es necesario tener una buena organización para lograr articular el TDNR y el TPR, lo cual implica aprovechar cada momento, regular el tiempo, tener horarios flexibles, y recibir "colaboración" en las tareas domésticas, compartiéndolas con la pareja, la familia o contar con personal doméstico contratado.

No obstante, son más frecuentes los factores que entorpecen esa "buena organización necesaria": la falta de priorización de las tareas (darle demasiada importancia a cada pequeña cosa), la falta de planificación (el dejar que las cosas sucedan), la soledad, la mayor demanda de los hijos cuando son pequeños, la decisión de llevar trabajo productivo al hogar, y la ausencia de horarios laborales fijos. Las mujeres vincularon como motivos que tienden a que esto ocurra a su estilo de vida, su forma de ser, su deseo de querer cumplir con las expectativas de todos, la dedicación de una mayor proporción de su tiempo al TPR que al TDNR, el deseo de hacer varias cosas al mismo tiempo, otorgarle mayor prioridad al TPR que a otros aspectos de su vida, y el cansancio que sienten como consecuencia de la doble jornada laboral.

"[...] lo voy resolviendo quizás espontáneamente, si obviamente con la jornada laboral no puedo decidir mucho, cumplo el horario y hago lo que tengo que hacer, pero después lo que tiene que ver con las tareas domésticas voy fijándome, si estoy muy cansada lo regulo pero no es algo que lo organice realmente [...]"

(31 años, profesional)

"[...] Trato de organizarme, pero sinceramente le dedico la mayor parte de mi tiempo al trabajo, aunque me gustaría tener más tiempo para mi casa, mis cosas [...]"

(33 años, profesional)

"[...] El tema es mi hijo, se lo tengo que dejar a alguien, se lo dejo a las abuelas, y eso es diariamente, vivir con una tensión, no lo puedo manejar yo. Me gustaría trabajar menos horas, por ahí tener alguien que me ayude $[\ldots] "$

(39 años, empleada)

(55 años, profesional) 
Cuidados de salud en las mujeres que tienen doble jornada laboral

$\mathrm{Al}$ analizar los cuidados de salud que realizan las mujeres entrevistadas, se observa que ellas consideran que, para llegar a un estado de bienestar y armonía, se deben complementar tres esferas distintas: la física, la social y la psicológica.

Con respecto a sus cuidados dentro de la esfera física mencionan la necesidad de realizar los controles de rutina indicados por su médico, como por ejemplo realizar el PAP, el control de la TA. Además, consideran importante el hecho de "conocerse", "quererse", "tener conciencia de su cuerpo", "recibir una buena alimentación", "no fumar" y "descansar bien". Dentro de la esfera psíquica, varias refieren realizar psicoterapia para sentirse saludables. Por último, en relación al aspecto social, les resulta importante llevar un estilo de vida saludable: hacer deportes, leer libros, pasar tiempo con la familia, realizar actividades de ocio, rodearse de buena gente y llevar una vida "sin excesos".

Muchas mujeres creen que antes, en el pasado, sus abuelas y madres se cuidaban menos y dejaban de lado la medicina, con tendencia a la auto-medicación con hierbas y recursos caseros. Atribuyen esto a la ausencia de controles preventivos o periódicos en otras épocas, con falta de información médica, menor acceso al sistema sanitario, ignorancia, menor comunicación y/o a la mayor dedicación al TDNR. En contraste, otras mujeres, principalmente las que vienen del interior del país, creen que sus abuelas y madres se cuidaban más, porque utilizaban remedios caseros de procedencia más natural, tenían una vida más saludable, más tiempo ocioso y menos estrés.

Volviendo a la actualidad, y en relación con la evolución de la medicina, las mujeres entrevistadas que valoran una medicina preventiva más desarrollada y la oportunidad de realizar más chequeos, perciben una dificultad en su acceso a la prevención a causa de falta de tiempo, más estrés y más situaciones conflictivas en su día a día, a pesar de tener mayor conciencia de enfermedades, el conocimiento de enfermedades más agresivas, mejor acceso a la información y mayor registro de sus cuerpos.

\section{"[...] No sé si se cuidaban.... No sé si tenían} tanta conciencia como nosotros ahora [...]"

(43 años, empleada)

"[...] Controlarse periódicamente, ir a la ginecóloga cada cuanto me diga ella; consultar si me encuentro algo. Estoy tratando de no fumar, pero me es casi imposible [...]"

(42 años, empleada)

"[...] Se auto-cuidaban menos, no creo que hayan ido al médico. Pero ahora nosotras no nos auto-cuidamos por falta de tiempo, no por falta de ganas [...]"

(30 años, profesional)

"[...] Es variable de acuerdo el medio en el que se hayan criado, era más difícil, había menos comunicación. Iban al médico cuando estaban de última, ahora como todo más medicina preventiva [...]"

(58 años, profesional)

\section{Recreación de las mujeres con doble jornada laboral}

En relación a los espacios de ocio, las mujeres entrevistadas expresaron que aprovechan los fines de semana y buscan, en ese tiempo, cortar con la vorágine de querer hacer todo, descansar la mente y el cuerpo. Hay un grupo pequeño de mujeres que manifestaron no tener ese espacio, porque siempre tienen alguna obligación por cumplir o tienen hijos pequeños que les demandan mucho tiempo. Enuncian esos espacios o actividades que les darían placer como viajar, ir al cine, teatro, hacer deportes, escuchar música, dormir, mirar televisión, caminar, andar en bicicleta, bailar, pintar, pasar tiempo con amigos y la familia. Para las mujeres mayores, estar en sus casas y "hacer nada" es su espacio de ocio, pero muchas viven ese "hacer nada" con sentimientos de culpabilidad.

"[...] Me refugio mucho en la literatura, en el cine, teatro, leo muchísimo. Disfruto muchísimo de las artes, de la música, mi hijo es sonidista. O sea, yo salgo de la escuela y meto la cabeza en el arte, veo muchas pinturas, voy a exposiciones todos los fines de semana, voy a todos los museos, a los de bellas artes, al Malba, al que sea [...]"

(56 años, profesional)

"[...] porque para cortar. Uno entró con una vorágine, con una desesperación de hacer todo, y de no llegar. Este, cortar con todo, incluso las obligaciones de la casa, no? Aunque esté la casa mugrienta, no importa, voy a leer un libro [...]"

(48 años, profesional)

"[...] Salir, ir al cine, no levantarme temprano, limpio dejo todo limpio y me voy a tomar mate, gozo de las simplezas de la vida, la mañana de sol, caminar me encanta [...]"

(51 años, profesional)

"[... justamente de eso, para no hacer nada, pero lo vivo con culpa, como todos los de mi generación [...]"

(58 años, profesional)

Disponibilidad para consultar al médico

Las mujeres que consultan con sus médicos para realizar controles de salud creen que es necesario tomarse el tiempo para hacerlo, pero aclaran que esto depende de la actitud de cada una y de la intención de querer cuidarse. Ellas, por lo general, refieren que tienen que tomarse un día o medio día laboral, y que siguen las indicaciones de sus médicos en cuanto a realizarse los estudios que les solicitan con motivos preventivos.

Aquellas que refieren no tener disponibilidad para consultar al médico lo adjudican a la falta de tiempo, horarios fijos poco flexibles en el ámbito laboral de su TPR, la preferencia por realizar otras actividades, o a que tienen que cancelar frecuentemente consultas agendadas porque les surgen obligaciones imprevistas. Entre estos motivos no aparece la influencia de las actividades domésticas (TDNR).

Por miedo a que se le diagnostiquen enfermedades, algunas mujeres refirieron tomar conductas que le permitan evitar la consulta médica cuando sea posible, como autoevaluarse y buscar información en Internet. 
"[...] Y se complica, si. Ahora por ejemplo tengo que hacer unos análisis y le estaba preguntando a la doctora si el sábado estará abierto el laboratorio. El sábado que se supone que es el día que uno descansa, que lava la ropa, que.. etc. $Y$ bueno yo lo tengo que ocupar con esto [...]"

(48 años, profesional)

"[...] mal porque no solamente las actividades domésticas sino lo que es trabajo, que no tengo horarios, no tengo horarios. Por ahí me cuesta hacérmelos mas que nada, por ahí tengo horarios, pero por ahí llego a mi casa y prefiero quedarme en mi casa ordenando o limpiando que ir al médico, eso sí, sin duda [...]"

(30 años, profesional)

"[...] más que nada con el trabajo, es como que tengo solo algunas mañanas libres $y$ ahí tengo que meter todas las consultas que quiero hacer $y$ cosas extras, porque termino de trabajar a las 8 y generalmente a esa hora ya no hay turnos [...]"

(32 años profesional)

Ambivalencias en cuanto a la exigencia percibida por la doble jornada laboral

A algunas de las mujeres entrevistadas el trabajar fuera del hogar (TPR) no les representa ninguna exigencia, ya que sienten que las relaja, les brinda sensación de armonía y salud. Por otro lado, otras perciben alteraciones en su estado de ánimo, o recibieron diagnóstico de ataques de pánico y/o depresión. Otro grupo de mujeres refiere que el TPR les genera falta de tiempo, competitividad profesional, problemas de salud generados por estrés, cansancio y dolores varios.

A modo de síntesis: el significado del trabajo

De la palabra de las entrevistadas se llega a una interpretación que permite la reflexión y significación del trabajo. Estas mujeres definen al trabajo (TPR) como una capacidad de independencia económica por la cual obtienen la gratificación para vivir; les da libertad, salud y placer. Las mujeres ven esa gratificación que les genera el TPR -además de la oportunidad de desarrollarse profesionalmente-, como parte de su dignidad, capacidad de obrar y de realizarse, tanto aquellas que aman su profesión, como quienes sienten que les genera una batalla diaria y un sacrificio por lo mal remunerado que consideran su trabajo. A otras les significa su cable a tierra, una necesidad emocional, un espacio para el desarrollo de su creatividad, realización de proyectos, comunicación y generación de relaciones interpersonales.

"[...] para mí el trabajo significa capacidad de obrar, sos una persona de derecho, una persona capaz y es todo, es no sé, realizarte. Sentirte realizada en la vida [...]"

(54 años, profesional)

"[...] El trabajo para mi es una parte más de la vida, no es lo más importante... ....algo que me gusta hacer, porque amo mi profesión. $Y$ a la vez también una forma, digamos, de ganar dinero [...] (Mujer de 33 años, universitaria) [...]Significa la manera de insertarse en el mundo de salir de nuevo y ser adulto $y$ después la supervivencia, es fundamental yo no puedo entender como una mujer se siente completa sin tener algo fuera del hogar, me cuesta entender pero sé que hay mujeres que están felices en ese rol, yo no tengo referencia de eso, mi mama siempre trabajo pero me parece una vida muy limitada [...]"

(41 años, profesional)

"[...] principalmente un sostén económico, igual creo que no podría dejar de trabajar, o sea hoy lo hago porq lo necesito para vivir, igual si fuera millonaria no creo que pudiera estar quieta en mi casa [...]"

(42 años, empleada por contrato)

"[...] Es vida, placentero, es salud, es lo que yo elegí [...]"

(43 años, empleada)

"[...] Significa mi vida, mis proyectos [...]"

(31 años, profesional)

\section{Discusión}

Debido a que, como noción, el trabajo permite diferentes miradas y análisis, según distintas disciplinas y trayectorias de pensamiento, la pregunta de partida a discutir podría haber sido ¿qué es el trabajo? Le seguirían: ¿qué es el trabajo para la mujer de clase media en el siglo XXI? y ¿cómo funciona la relación trabajosalud/cuidados de salud? ${ }^{18}$.

En este estudio se adoptó una mirada desde el género, pero de tinte sanitario. Se tomó como definición de "género" al conjunto de características psicológicas, sociales y culturales asignadas a mujeres y a hombres (aunque debería pensarse de carácter no binario), en cierto momento histórico, pasibles de transformaciones, que muestran las diferencias de acceso y de distribución del poder y de los recursos ${ }^{19}$. La asignación social de funciones y actividades ha naturalizado roles, y ha condicionado identidades y proyectos de vida. La naturalización de roles es uno de los principios organizativos de la vida social, y, de la auto-percepción de las trayectorias de vida y laborales. Adquiere un rol protagónico en las expectativas de los individuos, con estereotipos que cristalizan en competencias y tareas que condicionan las opciones profesionales para hombres y mujeres ${ }^{20}$. La deconstrucción de los estereotipos es un proceso lento y, a veces, contradictorio. Desde esta posición y en relación al tópico del estudio, el TPR -además de actividad económica-, es una vía de inclusión, de valoración social, representa una condición central para la autonomía, y por último, es un componente del proyecto de vida y de la autoafirmación de hombres y mujeres. No obstante, continúa siendo uno de los espacios generadores de inequidad ${ }^{21}$. Si bien la mujer se ha ido incorporando al mercado laboral, la división por género del trabajo la ha llevado a puestos alejados del poder, con tendencia a perpetuarla en ámbitos domésticos no remunerados o tareas poco valoradas. Se le ha adjudicado a las mujeres el trabajo TDNR, lo que resulta en inequidad, discriminación y segregación ocupacional ${ }^{22}$. En la actualidad, una nueva forma de discriminación implica que se le pida a las mujeres un perfil significativamente superior al de los varones para acceder a las mismas oportunidades laborales ${ }^{23}$.

Se completa el recorrido y los supuestos del equipo de trabajo con el otro pilar, la salud. Según el informe de Organización Mundial de la Salud (OMS) ${ }^{24}$ las mujeres viven en promedio seis a ocho años más que los hombres y realizan en general mayor cantidad de consultas al sistema por problemas de salud. 
La violencia y el abuso son temas prioritarios, y las enfermedades cardiovasculares, la principal causa de muerte en mujeres. Por otro lado, la mujer se enfrenta además los problemas reproductivos y su consiguiente influencia en la mortalidad materna que incluye la vinculada al aborto inseguro-, y también a la violencia de pareja. En relación a las enfermedades crónicas el cáncer del cuello uterino es el segundo tipo de cáncer más frecuente en la mujer, y prácticamente todos los casos están relacionados con la infección genital por papilomavirus humanos (VPH). A su vez las mujeres son más propensas que los hombres a la depresión y la ansiedad ${ }^{24}$, entidades asociadas a la sobrecarga laboral y/o a factores contextuales como los que éstas sufren por el mandato social de tener que ser las principales responsables del TDNR. Entonces, si se parte de una mirada sanitaria en el mapeo de las enfermedades según las diferentes etapas de la vida, cabe introducir cuestionamientos sobre la igualdad de oportunidades entre hombres y mujeres.

Respecto de la investigación que nos compete, podemos preguntarnos cómo contribuir al cuidado de la salud de las mujeres de clase media cuando éstas empiezan a trabajar fuera de su hogar, y cómo impacta la carga laboral horaria resultante de sumar el TPR y el TDNR.

De los resultados de este trabajo, se desprende de la voz de las mujeres la importancia de construirse autónomas, independientes, con proyectos, identidad y desarrollo profesional, lo cual destaca la importancia que tiene para las mujeres el acceso igualitario al TPR ${ }^{25}$. Cabe señalar además, que la ausencia de independencia económica las vuelve más vulnerables a situaciones de violencia de género.

Podríamos complejizar más aún el análisis si lo abriéramos a las problemáticas de las mujeres de menos recursos económicos, migrantes, trans, etc., lo que va más allá del alcance de esta investigación. Sin embargo, el diseño implementado no permite extrapolar resultados y conclusiones más allá del grupo de participantes seleccionadas, que se aproxima al tema considerando las experiencias de las mujeres entrevistadas. Estas mujeres, adultas, de clase media, la mayoría universitarias, con cobertura privada de salud resignifican positivamente el TP, pero aún siguen invisibilizando las tareas domésticas (TDNR), ya que muchas de ellas siguen mencionando la palabra "colaboración" cuando alguna otra persona de la familiar realiza un algún TPNR. Este modo de usar el lenguaje brinda evidencia de que ellas se sienten las principales responsables del TDNR.

Por otro lado, si bien algunas mujeres logran hacer visible la inequidad en la distribución del TDNR, sienten que no les queda otra alternativa que cumplir con la doble tarea ${ }^{26}$.

\section{Conclusiones}

Este trabajo permite documentar que las mujeres con TPR y TDNR valoran positivamente el primero, pero se cuestionan no dedicarle más tiempo al ámbito familiar. Por este motivo, resulta clara la auto-exigencia y la invisibilidad del TDNR, así como su distribución inequitativa.

En relación al auto-cuidado de su salud, sienten que no realizan los cuidados médicos que necesitan, a pesar de contar con buenos indicadores de prácticas preventivas recomendadas para las mujeres según su edad. Esto puede denotar una ausencia de reflexión acerca de que el TPR es parte de su "sentirse bien" y una sobrevaloración de la importancia de las consultas médicas preventivas.

Fuente de financiamiento/Conflicto de interés de las autoras: El presente trabajo de investigación fue autofinanciado por las investigadoras, quienes declaran no tener conflictos de interés para la realización de este estudio.

\section{Agradecimientos}

A Natalia Rosli y Mariela Weisbrot, quienes realizaron la revisión de pares del manuscrito.

A Laura Massaro, por otorgar autorización para emplear su obra 'Aquí estamos' como imagen de portada.

\section{Referencias}

1. Scott J. La mujer trabajadora en el siglo XIX. In: Duby G, Perrot M, et al., editors. Historia de las mujeres. Madrid: Taurus; 1993.

2. Rogerat C, Maruani M, Torns T. Las nuevas fronteras de la desigualdad: hombres y mujeres en el mercado de trabajo. Barcelona: Icaria Antrazyt, $156 ; 2000$.

3. Bock G, Thane P. Maternidad y políticas de género: la mujer en los estados de bienestar europeos, 1880-1950. España: Cátedra; 1996.

4. Lewis J. Women in England, 1870-1950: sexual divisions and social change. UK: Wheatsheaf Books; 1984.

5. Huberman H, and LT. Masculinidades Plurales. Reflexionar en clave de géneros. 1st ed. Buenos Aires, Argentina: Programa Naciones Unidas para el Desarrollo; 2012.

6. Rodríguez A. Del Reparto Del Empleo Al Reparto Del Trabajo. In: and others, editor. El Futuro del trabajo. Reorganizar y repartir desde la perspectiva de las mujeres. España; 1996.

7. Wainerman C. Mujeres que trabajan. Hechos e ideas. In: Torrado S, editor. Población y bienestar en la Argentina. Del primero al segundo centenario. Estudios del Bicentenario. Buenos Aires: Edhasa; 2007

8. Faur E. Género, masculinidades y políticas de conciliación familia-trabajo . Nómadas (Col). 2006;24:130-141. Available from: http://nomadas.ucentral.edu.co/index.php/inicio/25-genero-y-politicas-publicas-desafios-de-la-equidad-nomadas-24/340-genero-masculinidadesy-conciliacion-familia-trabajo.

9. Lan D. Doble jornada laboral e invisibilidad del trabajo de las mujeres. Notas a partir de un caso. In: and others, editor. $5^{\circ}$ Congreso Nacional de Estudios del Trabajo. Pre -Congreso FCH-UNICEN; 2001.Available from: https://geografiadegeneroargentina.files.wordpress.com/2017/06/landianaaset-2001.pdf.

10. Federici S. Revolución en punto cero. Trabajo doméstico, reproducción y luchas feministas. vol. 13. Madrid, España: Traficantes de Sueños; 2013. p. $978-84$.

11. Denzin N, Lincoln Y. Manual de investigación cualitativa Vol 1: El campo de la investigación cualitativa. vol. 1. and others, editor. España: Gedisa; 2012.

12. Vasilachis-De-Gialdino SA. Estrategias de investigación cualitativa. España: Gedisa; 2006.

13. Glaser BG, and ALS. The Discovery of Grounded Theory: Strategies for Qualitative Research. 7th ed. USA; 2012.

14. Denzin N, Lincoln Y. Manual de investigación cualitativa. Vol 3: Las estrategias de investigación cualitativa. España: Gedisa; 2012.

15. Cáceres P. Análisis cualitativo de contenido:una alternativa metodológica alcanzable. . Psicoperspectivas Revista de la Escuela de Psicología Facultad de Filosofía y Educación Pontificia Universidad Católica de Valparaíso. 2003;Il:53-82. Available from: 10.5027/psicoperspectivas-vol2issue1-fulltext-3.

16. Schettini P, Cortazzo I. Análisis de datos cualitativos en la investigación social. Procedimientos y herramientas para la interpretación de información cualitativa. Libros de Cátedra. La Plata, Argentina: Editorial de la Universidad Nacional de La Plata (EDULP); 2015.

17. Garay OE. Consentimiento informado: antecedentes éticos y jurídicos; 2017. Available from: http://www.salud.gob.ar/dels/entradas/consentimientoinformado-antecedentes-eticos-y-juridicos [Last access: 2020-04-20]. 
18. Aldonate AE. Las mujeres y el mundo del trabajo en la Argentina de la primera mitad del Siglo XX. Bernal, Argentina: Repositorio Institucional Digital de Acceso Abierto de la Universidad Nacional de Quilmes; 2015. Available from: http://ridaa.unq.edu.ar/handle/20.500.11807/62.

19. Gamba S, Diz T. Diccionario de estudios de género y feminismos. Buenos Aires, Argentina: Biblos; 2007.

20. Programa de las Naciones Unidas para el Desarrollo. Aportes para el DH en Argentina. Género en el trabajo: brechas en el acceso a puestos de decisión. . Buenos Aires, Argentina: Programa de las Naciones Unidas para el Desarrollo.; 2014. Available from: https://www.undp.org/content/dam/ argentina/Publications/Desarrollo\%20Humano/PNUD\%20Argentina\%20S\%C3\%ADntesis\%20PrensaAPortes\%208.pdf.

21. González-Gómez MF. Salud laboral y género. Apuntes para la incorporación de la perspectiva de género en el ámbito de la prevención de riesgos laborales. Med segur trab. 2011;57(1):89-114. Available from: 10.4321/S0465-546X2011000500007.

22. Buedo-Martínez S. Mujeres y mercado laboral en la actualidad, un análisis desde la perspectiva de género: Genéricamente empobrecidas, patriarcalmente desiguales. Revista de educación social. 2015;21. Available from: http://www.eduso.net/res/21/articulo/mujeres-y-mercado-laboral-en-laactualidad-un-analisis-desde-la-perspectiva-de-genero-genericamente-empobrecidas-patriarcalmente-desiguales.

23. Henrich I, et al. Nuevas problemáticas de género y desigualdad en América Latina y el Caribe. and others, editor. Ciudad Autónoma de Buenos Aires, Argentina: CLACSO; 2017. Available from: http://biblioteca.clacso.edu.ar/clacso/se/20170313031536/NuevasProblematicasDeGeneroyDesigualdad. pdf.

24. Organización Mundial de la Salud. Salud de la mujer; 2018. Available from: https://www.who.int/es/news-room/fact-sheets/detail/women-s-health [Last access: 2020-07-01]

25. Noriega M, Gutiérrez G, Méndez I, et al. Las trabajadoras de la salud: vida, trabajo y trastornos mentales. Cadernos de Saúde Pública. 2004;20(5):1361-1372. Available from: 10.1590/s0102-311x2004000500031; https://dx.doi.org/10.1590/s0102-311x2004000500031.

26. Nieves-Rico M, Marco F. Mujer y empleo: la reforma de la salud y la salud de la reforma en Argentina. 1st ed. Buenos Aires: Siglo Veintiuno, CEPAL 2006. Available from: https://repositorio.cepal.org/bitstream/handle/11362/1949/1/S3314R541_es.pdf.

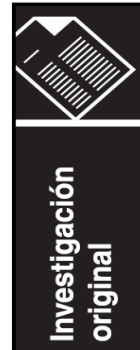

